\title{
Side-coupled slab-symmetric structure for high-gradient acceleration using terahertz power
}

\author{
R. B. Yoder* and J. B. Rosenzweig \\ Department of Physics and Astronomy, University of California, Los Angeles, \\ 405 Hilgard Avenue, Los Angeles, California 90095-1547, USA \\ (Received 20 November 2004; revised manuscript received 25 October 2005; published 17 November 2005)
}

\begin{abstract}
A slab-symmetric dielectric-loaded accelerator structure, consisting of a vacuum gap between dielectric-lined conducting walls, is described. The device is resonantly excited by an external drive laser which is side coupled into the acceleration region; a novel coupling scheme, which consists of an array of narrow, equally spaced slots in the upper structure boundary, is presented and analyzed in detail. This structure partakes of the advantages of earlier slab-symmetric optical acceleration proposals, but will use a terahertz-frequency external radiation source $(\lambda=340 \mu \mathrm{m})$, allowing realistic electron beams to be used in a proof-of-principle experiment. Two- and three-dimensional electromagnetic simulations are used to verify the mode patterns and study the effects of the couplers, including time-dependent calculations of the filling of the structure and particle-in-cell computations of the beam wakefields. Details of the resonance are found to be highly sensitive to the coupling slot geometry: the presence of the couplers can lead to frequency detuning, changes in the field breakdown limits and overall $Q$ factor, and distortions of the field pattern. Beam wakefields are enhanced by the presence of the slots, but found to have no significant effect on the beam transport. The resonant accelerating fields, which are nearly constant along the short transverse direction, are found to have between 10 and 15 times the amplitude of the driving radiation, with only a small $(<10 \%)$ admixture of other nonaccelerating modes. Field gradients are computed to be near $100 \mathrm{MV} / \mathrm{m}$ when the structure is driven with $100 \mathrm{MW}$ of terahertz laser power. Possible manufacturing methods for a prototype device are discussed.
\end{abstract}

DOI: $10.1103 /$ PhysRevSTAB.8.111301

PACS numbers: 41.75.Jv, 41.20.Jb, 42.62.-b

\section{INTRODUCTION}

Laser-powered accelerator structures have often been proposed as a possible component of a next-generation accelerator capable of producing the extremely highbrightness electron and positron beams demanded by high-energy physics experiments. Interest in laser-driven acceleration has remained high over the past decade, inspired by the demonstration of ever more sophisticated and powerful laser sources that can achieve large energy densities at a reasonable cost. In all such concepts, the large transverse fields of a laser pulse must be converted into useful longitudinal fields. The difficulties of using traditional cavitylike structures for this purpose are familiar, including large surface fields (tending to cause electric breakdown) and the deleterious effects of wakefields on high-charge accelerating beams.

Among the alternate strategies that have been considered for laser acceleration is the use of a nontraditional, translationally symmetric geometry for the resonant structure, rather than the conventional cylindrical symmetry [1]. Such a "slab geometry" avoids many experimental difficulties inherent in other common approaches - the need to produce and control a plasma disturbance (as required for, e.g., the plasma beat-wave accelerator [2]), or the inefficiency and high transverse field components inherent in

\footnotetext{
*Present address: Dept. of Physics, Manhattan College, Riverdale, NY 10471.

Email address: ryoder@mailaps.org
}

accelerators which use freely propagating laser fields directly (such as the inverse Cerenkov accelerator [3] and crossed-beam vacuum accelerators [4,5]). The advantageous properties of slab symmetry have been discussed elsewhere (e.g. $[1,6]$ ) and include the strong suppression of transverse beam wakefields, lack of higher-order synchronous modes, mitigation of field breakdown via the use of dielectric materials, and acceleration of high-charge beams without significant beam loading. With a resonant structure, peak accelerating fields may be much higher than those of the drive laser.

The basic theory of acceleration and wakefields in a slab-symmetric resonant structure has been presented and discussed in several papers over the last decade [1,6-8], together with some preliminary versions of an accelerator at optical wavelengths (specifically $\lambda=10.6 \mu \mathrm{m}$ ). In this paper, we describe a similar slab-symmetric accelerator at terahertz frequencies $(\lambda=340 \mu \mathrm{m})$ and devote particular attention to the details of coupling power into the device, using a novel design which relies on a series of transverse slots. To our knowledge, only one previous paper [9] has attempted a detailed coupling analysis of a laser-driven accelerator; the results presented here indicate that failure to consider coupling methods may be a serious omission. (Other types of dielectric-loaded slab structures have also been proposed for wakefield acceleration [10-12] and tested in experiment [13]. A theoretical analysis of wakefield acceleration shows that a very large number of structure modes are produced by the initial driving bunch, some of which combine to produce useful accelerating fields 
behind the bunch [14,15]; in contrast, the resonant design discussed here operates in a single dominant mode.)

\section{STRUCTURE GEOMETRY AND FIELD THEORY}

Fundamentally, this slab-symmetric accelerator consists of a pair of parallel dielectric planes, separated by a narrow vacuum gap and bounded above and below by a thin conductive layer. Periodic slots in the conductor provide a means for coupling radiation into the gap and also enforce longitudinal periodicity in the structure fields; without such modulation of the structure, the fields would consist of $z$-independent Fabry-Perot modes. On the other hand, when longitudinal periodicity is added and the correct resonant geometry is achieved, the mode pattern is dominated by a longitudinal standing wave having a phase velocity exactly equal to the speed of light. The array of slots extends throughout the interaction length (defined as, roughly, the diameter of the laser spot impinging on the upper surface). The concept is illustrated in Fig. 1.

Any periodic modulation in the coupling or dielectric properties of the structure would serve to generate usable accelerating fields, and previous approaches to this design have each taken a different approach to generating this periodicity $[1,7,8]$. (A related idea is used in the Braggreflector accelerator recently analyzed in Ref. [9].) The periodic slots used here represent the optimum choice for coupling power preferentially into the accelerating mode. However, they significantly perturb the resonant frequency of the structure, as will be discussed below, and their effect on the fields must be included in the design. Coupling slots also appear on the lower surface, as shown in the figure; these serve to symmetrize the design and also allow the use of transmitted radiation as a diagnostic. Designs which, like this one, "leak" power from the accelerating mode have been proposed in several other contexts (e.g. [16,17], both of which are discussed briefly below).

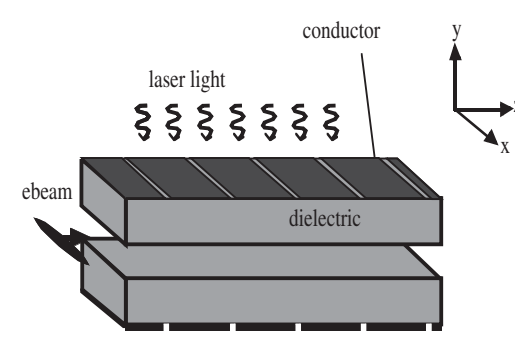

(a) (b)

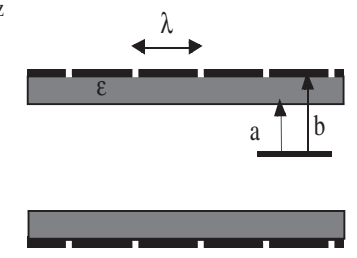

FIG. 1. (a) Schematic drawing of the structure geometry. Two layers of dielectric-lined conductor surround a vacuum gap; a very wide electron beam is injected into the gap and travels in the $+z$ direction, while radiation (polarized in $z$ ) is coupled in from above through transverse slots in the conductor. (b) A cross-section in $x$, showing the parameters used in the analysis.

\section{A. Summary of analytic mode theory}

The accelerating-mode fields for a structure with infinite transverse extent are straightforward to derive and have been presented in e.g. [7]; the effects of a finite transverse dimension on beam stability have been considered in [6]. These results will be briefly summarized here. For the purposes of our discussion, we use the axes and structure parameters defined in Fig. 1, so that the vacuum gap has width $2 a$ and the structure itself width $2 b$, with dielectric (of relative permittivity $\epsilon$ ) occupying the regions from $|y|=a$ to $|y|=b$. The structure is illuminated from the $+y$ direction by a laser polarized in the $z$ direction, with beam propagation along the $z$ axis. We assume infinite extent in the $x$ dimension and consider only synchronous (speed-of-light) accelerating fields, equivalent to taking the field periodicity in $z$ equal to the free-space laser wavelength. The dispersion relation then enforces $k_{x}=k_{y}=0$ within the vacuum gap $(|y|<a)$; the solution for $E_{z}$ is therefore independent of $y$ and we have

$$
\begin{gathered}
E_{z}=E_{0} \cos \left(k_{z} z\right) e^{i \omega t}, \\
E_{y}=E_{0} k_{z} y \sin \left(k_{z} z\right) e^{i \omega t},
\end{gathered}
$$

in the vacuum region and

$$
\begin{gathered}
E_{z}=A E_{0} \sin \left[k_{z} \sqrt{\epsilon-1}(b-y)\right] \cos \left(k_{z} z\right) e^{i \omega t}, \\
E_{y}=-\frac{A E_{0}}{\sqrt{\epsilon-1}} \cos \left[k_{z} \sqrt{\epsilon-1}(b-y)\right] \sin \left(k_{z} z\right) e^{i \omega t}
\end{gathered}
$$

within the dielectric. The allowed wave numbers $k_{z, n}$ are the roots of

$$
k_{z, n} a \frac{\sqrt{\epsilon-1}}{\epsilon}=\cot \left[k_{z, n} \sqrt{\epsilon-1}(b-a)\right]
$$

and the relative amplitude of the electric field in the dielectric is given by

$$
A=\csc \left[\sqrt{\epsilon-1}(b-a) k_{z, n}\right] .
$$

In practice, the $n=1$ fundamental mode gives the optimal field configuration. We note that Steinhauer and Kimura [16] have recently obtained an equation similar to (5) to describe a speed-of-light mode able to propagate in a slabsymmetric metal waveguide. Their structure, which exploits the finite penetration of the accelerating field into the metal wall at infrared wavelengths, displays some similar physics to the present work, since the metal essentially plays a role analogous to that of the dielectric liner.

The accelerating modes of a physically realizable structure, one which is very wide in the horizontal dimension but not infinite, were first investigated in [6]. When illuminated with a circular Gaussian laser pulse of finite-width $\sigma_{r}$, the accelerating fields in an open structure will tend to fall off in the $x$ dimension like $\exp \left(-x^{2} / \sigma_{r}^{2}\right)$. To find an approximate analytic field solution near the center of the 
finite structure, one may assume conducting boundaries at $x= \pm L$, where $L \gg b$. The eigensolutions then exhibit a cosine form for the field dependence on $x$, with associated wave vector $k_{x}$; taking $k_{x} L=\pi / 2$, the field near $x=0$ has the same limiting form as a Gaussian, giving an estimate of the resulting variation of $E_{z}$ as a function of $y$.

For synchronous accelerating fields $\left(\omega=c k_{z}\right.$ in the vacuum gap), the dispersion relation requires a hyperbolic variation in the $y$ dimension, i.e. $k_{y}=i k_{x}$. Thus the acceleration fields have the form

$$
E_{z}(x, y, z)=E_{0}\left\{\begin{array}{c}
\cosh \left(k_{x} y\right) \\
\sinh \left(k_{x} y\right)
\end{array}\right\} \cos \left(k_{x} x\right) \cos \left(k_{z} z\right) e^{i \omega t},
$$

where the upper and lower terms represent even and odd solutions, respectively, and where $k_{x}=\pi / 2 L$. The full field solution gives a hybrid (HEM) mode with nonvanishing (though small) TE component.

Using the symmetric solution, the eigenvalue equation for $k_{z, n}$ becomes

$$
\begin{aligned}
(1+\epsilon) k_{x}^{2}+(1-\epsilon) k_{z, n}^{2}= & -k_{x} \xi_{n}\left\{\epsilon \operatorname{coth}\left(k_{x} a\right) \cot \left[\xi_{n}(b-a)\right]\right. \\
& \left.+\tanh \left(k_{x} a\right) \tan \left[\xi_{n}(b-a)\right]\right\},
\end{aligned}
$$

where $\xi_{n}=\sqrt{(\epsilon-1) k_{z, n}^{2}-k_{x}^{2}}$. Note that this equation reduces to Eq. (5) as $k_{x} \rightarrow 0$.

In a realistic experimental situation, the laser pulse width $\sigma_{r}$ will naturally be much greater than the structure dimension $a$, so that $k_{x} \ll k_{y}$. The quantity of interest for the accelerating fields is the resultant amount of nonuniformity of $E_{z}$ within the vacuum gap, i.e., the value of $E_{z}(y=a) / E_{z}(y=0)=\cosh \left(k_{x} a\right)$. For a laser spot size of $10 \lambda$, roughly the smallest possible for terahertz radiation, we have $k_{x} \approx 1 / \sigma_{r} \approx 300 \mathrm{~m}^{-1}$, and $\cosh \left(k_{x} a\right)=1.0006$. The magnitude of $E_{x}$ is likewise of order $10^{-4} E_{z}$. An aspect ratio of $L / a \approx 30$ is thus clearly sufficient to produce a good approximation to the infinite-slab fields.

Longitudinal and transverse wakefields generated by a ribbon beam in a finite structure have been calculated analytically in [6], showing good agreement with numerical simulation. Instability growth due to wakefields of a finite-width beam was also considered in detail. It was shown that, though both the flute and multibunch beam breakup instabilities may occur, they grow far more slowly than in comparable cylindrical geometries, and may be controlled by relatively straightforward means, including vertical focusing and detuning methods.

\section{B. Coupling to the structure}

The coupling slots which enforce the field periodicity as well as allowing radiation into the structure are an effective way to drive the accelerating mode efficiently, but in contrast to the usual waveguide couplers employed with microwave accelerating cavities, these slots have a transverse width which is effectively infinite, and hence they are not cut off for the relevant radiation frequency. They will thus inevitably be filled with field as the structure itself fills, with two consequences: first, there are unavoidably large fields within the slots, which become the most likely electric breakdown site, and second, the resonant frequency of the structure will be perturbed by the slots.

As a way to understand the behavior of the slot fields and estimate their magnitude, we can consider each slot to represent a parallel-plate transmission line of length $\Lambda$ equal to the thickness of the structure's metal wall (as illustrated in Fig. 2). The line is driven by wall currents on the inner surface of the structure boundaries and terminates in an open circuit at the outer surface. This strategy assumes that the slot width $w$ is small compared to the radiation wavelength $\lambda_{0}$, implying that the $z$-directed field within the slot can be taken as approximately constant.

Fields within the slots then have the form

$$
\begin{gathered}
E_{z}(y, t)=2 E_{0} \cos \left[k_{g}(\Lambda-y+b)\right] e^{i \omega t-i k_{g}(\Lambda+b)}, \\
H_{x}(y, t)=2 i E_{0}\left(\sqrt{\epsilon} / \eta_{0}\right) \sin \left[k_{g}(\Lambda-y+b)\right] e^{i \omega t-i k_{g}(\Lambda+b)},
\end{gathered}
$$

where the slot extends from $y=b$ to $y=b+\Lambda, \eta_{0}$ is the impedance of free space, and $k_{g}=2 \pi / \lambda_{g}$, with the guide wavelength $\lambda_{g}=\lambda_{0} / \sqrt{\epsilon} . E_{0}$, the excitation amplitude of the propagating waves, can be estimated from the wall current $J_{x}$ associated with the accelerating field in the structure; we note that $E_{0}$ will be approximately inversely proportional to $\epsilon$, since it effectively arises from the field generated by a time-varying charge distribution at $y=b$. Note that setting $\Lambda=\lambda_{g} / 4$ results in a quarter-wave matching condition in which the slot fields vanish at $y=$ $b$, the inner surface; the structure fields are then unper-
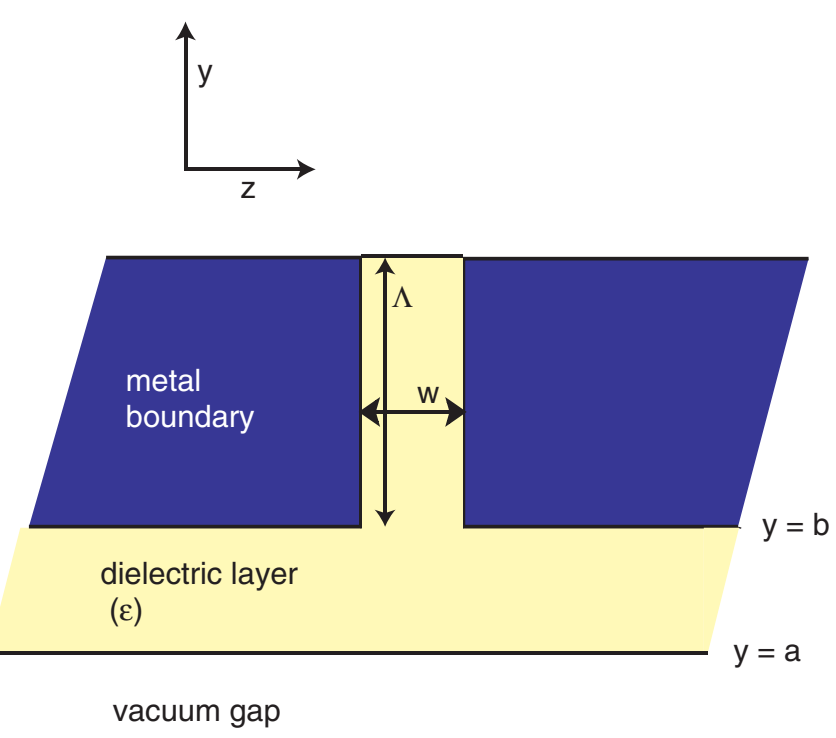

FIG. 2. (Color) Detail of coupling slot geometry, showing parameters used in the analysis. 
turbed by the presence of the slots. We will show below that simulation of the structure filling under these conditions confirms this behavior, with very long filling times, i.e., large values for the coupling $Q$ factor.

To estimate the perturbation of the resonant frequency by the presence of the slot fields, we can employ a version of the Slater perturbation theorem, which states that for small volume changes in a resonant structure, the frequency shift can be found from the relative change in magnetic and electric field energy. For the slab structure with slots, we have

$$
\frac{\Delta \omega}{\omega_{0}}=\frac{\int_{\text {slots }}\left(\mu|H|^{2}-\epsilon|E|^{2}\right) d V}{\int_{y<b}\left(\mu|H|^{2}+\epsilon|E|^{2}\right) d V},
$$

where the fields in the numerator are given by Eqs. (9) and (10) and in the denominator by the unperturbed structure fields, Eqs. (1)-(4). We implicitly assume that the field energy in the slots is small compared to the total field energy of the structure, which will be true for sufficiently narrow slots, and ignore the perturbation of the structure fields by the slots. Explicit calculation gives the result

$$
\frac{\Delta \omega}{\omega_{0}}=\frac{-4 \lambda_{0}\left(\sin 2 k_{g} \Lambda\right) / k_{g} w}{\pi^{4}\left[a \sin ^{2} \zeta+\frac{2}{3} \epsilon a \cos \zeta+(b-a) \epsilon\right]},
$$

in which $\zeta=k_{z} \sqrt{\epsilon-1}(b-a)$. Equation (12) predicts that for small $w$ and $\Lambda$ the frequency detuning should be proportional to $\Lambda / w$, and that there should be no detuning effect for $\Lambda=\pi / 2 k_{g}$, as mentioned previously. Reasonable agreement with simulation is found for small values of $w$.

\section{All-dielectric structure}

A structure without metallic boundaries would be advantageous at shorter wavelengths, when the thickness of the outer conductive layer could become less than a skin depth. One approach for scaling the slab-symmetric resonant structure to near-optical frequencies is the replacement of the metal boundary by a dielectric multilayer (or Bragg reflector) arrangement. This approach leads in essence to a one-dimensional photonic band gap accelerating structure, which is similar to the optical Bragg structures recently analyzed by Mizrahi and Schächter [17] and by Zhang and colleagues [9]. (The use of side-coupling slots to fill the structure with laser power in our device contrasts with the treatment in [17], in which power is coupled in along the beam axis. Our structure is also "leaky" by design, since a small amount of power will exit through the slots on both upper and lower surfaces, as discussed above. In [17], a speed-of-light mode would be perfectly confined within the structure in the ideal infinite case, and the confinement in a finite Bragg reflector can be made arbitrarily good through correct choice of geometry. Our design also differs significantly from the approach taken in [9], where the side coupling is not itself modulated, but a grating is used within the structure to maintain selective mode excitation.) Initial versions of an all-dielectric design have been presented elsewhere [18]; while details of these structures are outside the scope of this paper, simulation results indicate that a $10.6-\mu \mathrm{m}$ resonator can be constructed with a nine-layer Bragg reflector replacing the metal boundaries. After adjustment of the values of $a$ and $b$, accelerating field amplitudes on the order of 5 to 10 times those of the driving radiation can be achieved; however, the nonaccelerating Fabry-Perot mode has comparable amplitude and will be difficult to suppress entirely. Nevertheless, the much higher laser power available at short wavelength would still allow extremely high accelerating gradients.

\section{SIMULATION RESULTS}

Simulation of the slab-symmetric structures is difficult to achieve in full generality, since the varying length scales, unusual boundary conditions, and periodic nature of the structure are poorly accommodated by standard codes. The results described here include both eigensolutions and time-domain fields, but are necessarily approximations to the full time-dependence and three-dimensional nature of the structure behavior.

\section{A. Structure eigenmodes}

Eigensolutions for the fields of the terahertz infinitewidth slab structure, in the absence of coupling slots, were calculated using the three-dimensional (3D) finitedifference code GDFIDL [19]. These simulation results are qualitatively similar to those already demonstrated (via 2D simulation) for smaller structures [7], but they also allow estimation of numeric parameters such as the shunt impedance. In order to adapt the geometry for a 3D model, the simulation region was taken to be $2 \mathrm{~mm}$ wide in the $x$ dimension, with magnetic boundary conditions applied at $x= \pm 1 \mathrm{~mm}$ in order to obtain a nearly constant field in the wide dimension without requiring a prohibitively large computational volume.

If the outer conducting layers of the accelerator (see Fig. 1) are taken to be copper, with appropriate values for conductivity and losses, the structure ohmic $Q$ is calculated to be approximately 600 , and the simulated fields give rise to a shunt impedance for the structure of $15.3 \mathrm{M} \Omega / \mathrm{m}$. This relatively low value is a consequence of the slab geometry, which may be understood in this context as a combination of a large number of cylindrically symmetric structures in parallel.

\section{B. Beam wakefields}

The vanishing of transverse wakefields in a smooth slabsymmetric structure, using an infinitely wide beam, has been verified using simulation in e.g. $[7,18]$. Longitudinal wakefields under those conditions are negligible for an 
electron bunch length on the order of $\lambda_{0}$. However, one might reasonably expect the presence of the coupling slots to modify (and possibly strengthen) the wakefields, due to the passage of the electron beam near what is essentially a diffraction grating. Under these conditions, coherent radiation can be produced via the Smith-Purcell effect [20,21], with the relation

$$
\lambda=\frac{\ell}{|n|}(1 / \beta-\cos \theta)
$$

connecting radiation wavelength $\lambda$, grating period $\ell$, diffraction order $n$, electron relative velocity $\beta$, and emission angle $\theta$. As $\beta \rightarrow 1$ for a relativistic beam, the emission angle $\theta \rightarrow 1 / \gamma$, as usual, and $\lambda$ becomes much less than $\ell$. We therefore expect the Smith-Purcell radiation to be dominated by wavelengths in the nanometer range; further, the angular power distribution of forward radiation for a relativistic beam (discussed in [21]) implies that such radiation is exponentially suppressed unless $4 \pi x_{0} / \ell \approx$ $1 / \gamma$, where $x_{0}$ is the offset of the electron beam from the grating. For the $340-\mu \mathrm{m}$ slot-coupled accelerator discussed here, the left and right sides of this relation differ by nearly 2 orders of magnitude. Suppression of forward radiation implies a negligible interaction of the beam with its own wakefields; nevertheless, the form of the wakefields behind the bunch will in general be altered by the presence of the slots, due to the presence of a confined (nonsynchronous) grating mode near the surface.

To quantify these additional effects, we have simulated the passage of a ribbon electron beam through 17 periods of a slotted structure using the 2D particle-in-cell code OOPIC [22,23]. Comparing the longitudinal (retarding) wakefields with and without slots, we see some small change in the peak field magnitude, though the field pattern becomes less regular, as shown in Fig. 3. A structure resonant at $\lambda_{0}=340 \mu \mathrm{m}$ driven by a beam having $\sigma_{z}=$ $30 \mu \mathrm{m}$ has a retarding wakefield of only $10 \mathrm{kV} / \mathrm{m}$, as shown in Fig. 4. Different admixtures of modes are clearly visible in the figure.

The transverse wakefield cancellation, while exact in the unslotted case, becomes a partial suppression in the slotted structure. Transverse fields of up to $20 \mathrm{kV} / \mathrm{m}$ exist within the structure, whereas fields in the $1 \mathrm{MV} / \mathrm{m}$ range are found within the dielectric; details of the field shape depend on the slot geometry. In general, the structure resembles a grating less and less as the slot width and depth are increased, causing the transverse fields in the vacuum gap to decrease slowly. Figure 4 compares the resulting transverse wakes for several slot widths.

\section{2D Filling and Slot Effects}

Simulation of the time-dependent filling of the structure through the coupling slots has been demonstrated using both GDFIDL and a custom two-dimensional finitedifference code [7]. The theoretical dependence of the
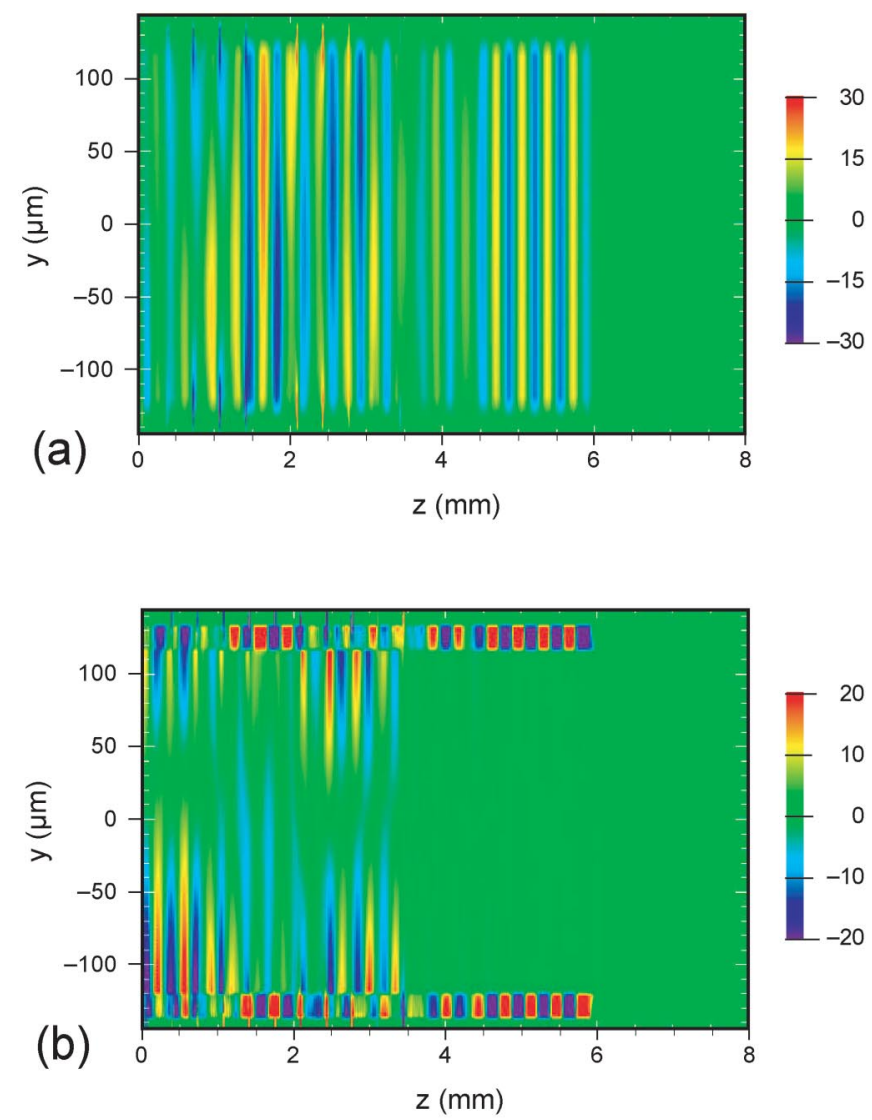

FIG. 3. (Color) False-color plots (from OOPIC simulation) of wakefields behind an infinitely wide electron beam passing through a slab-symmetric structure with an array of coupling slots extending the length of the structure. Structure dimensions are as given in the right-hand column of Table I; the (cold) electron beam has $\sigma_{z}=100 \mu \mathrm{m}, \sigma_{r}=30 \mu \mathrm{m}$, is located at $z=$ $5.8 \mathrm{~mm}$ and is moving in the $+z$ direction. (a) Longitudinal wakefields $\left(E_{z}\right)$, in $\mathrm{kV} / \mathrm{m}$. (b) Transverse wakefields, $E_{y}-c B_{z}$, in $\mathrm{kV} / \mathrm{m}$. Note that wakefields within the dielectric are saturated; they are generally at least an order of magnitude larger than those in the vacuum gap.

frequency perturbation on slot dimensions agrees approximately with simulation for small slot depths, though frequency resolution is limited by the simulation grid size. Further, the use of quarter-wavelength matching in the slot length to give unperturbed structure fields was verified, as shown in Fig. 5(a), where a two-dimensional calculation shows strong fields in the slots that have no effect on acceleration fields when the slot length $\Lambda$ is approximately $\lambda_{g} / 4$. In the same figure, these fields are compared with those of a structure with 5- $\mu \mathrm{m}$ coupling slots, much shorter than $\lambda_{g} / 4$. For this second case, the accelerating field is deformed in the vicinity of the slot and the resonant frequency is increased.

Figure 6 compares the relative amplitudes of the accelerating and Fabry-Perot modes for these two structures (detailed parameters of which are presented in Table I). Clearly, the quarter-wavelength slots give a structure with 
(a)

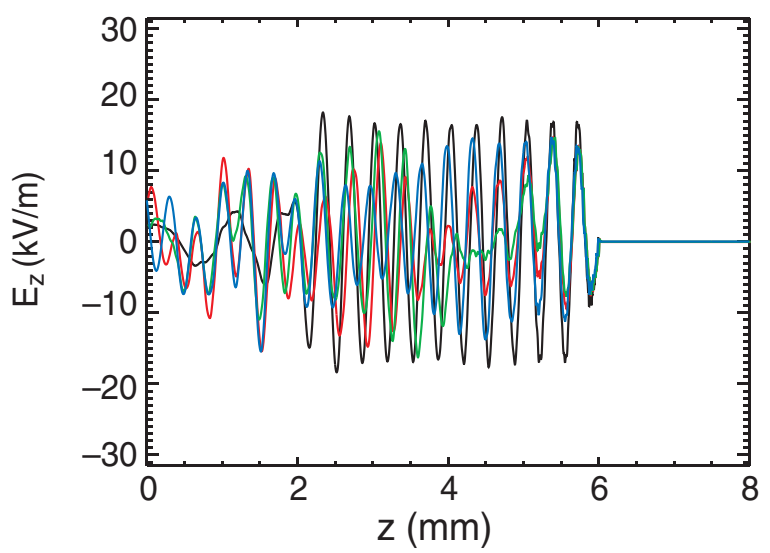

(b)

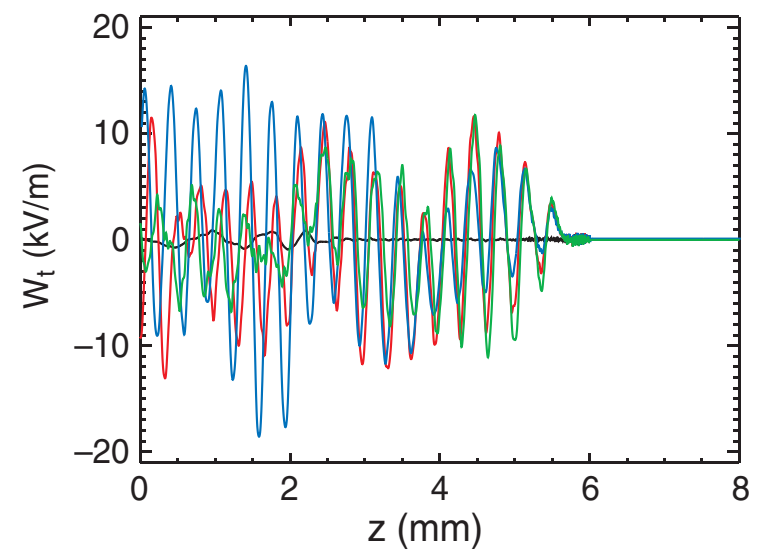

FIG. 4. (Color) Lineouts of beam wakefields as a function of axial position, for the same fixed structure geometry and electron beam parameters as in Fig. 3 but with varying slot width, as follows: black, no slots; blue, $10 \mu \mathrm{m}$, red, $20 \mu \mathrm{m}$, green, $30 \mu \mathrm{m}$. (a) Longitudinal wakefields $E_{z}$, along the $z$ axis. (b) Transverse wakefields $W_{t}=E_{y}-c B_{z}$, along the line $x=0, y=$ $+33 \mu \mathrm{m}$.

high $Q$ factor-the accelerating field amplitude is more than 15 times that of the drive laser - with a concomitant long filling time (more than half a nanosecond). Likewise, the structure shows excellent mode quality, as the competing Fabry-Perot mode has less than one-half percent the amplitude of the accelerating mode. However, the field strength within the slots is also very large - at the outer end, more than 3 times the peak accelerating field. The $5-\mu \mathrm{m}$ slots lead to a significantly reduced $Q$ factor and field enhancement, with noticeably poorer mode quality, but the field in the slot is in general less than the peak accelerating fields. (There will, however, be relatively large surface fields at the inner and outer corners of the slot. While the field magnitude in the slot center is at most 0.4 times the accelerating field amplitude $E_{\max }$, there are also field spikes at the outer slot aperture with magnitudes that are sensitive to the simulation grid but are on the order of $1.3 E_{\max }$ to $1.5 E_{\max }$. The slot corners will hence remain the most likely breakdown location.) Furthermore, the filling

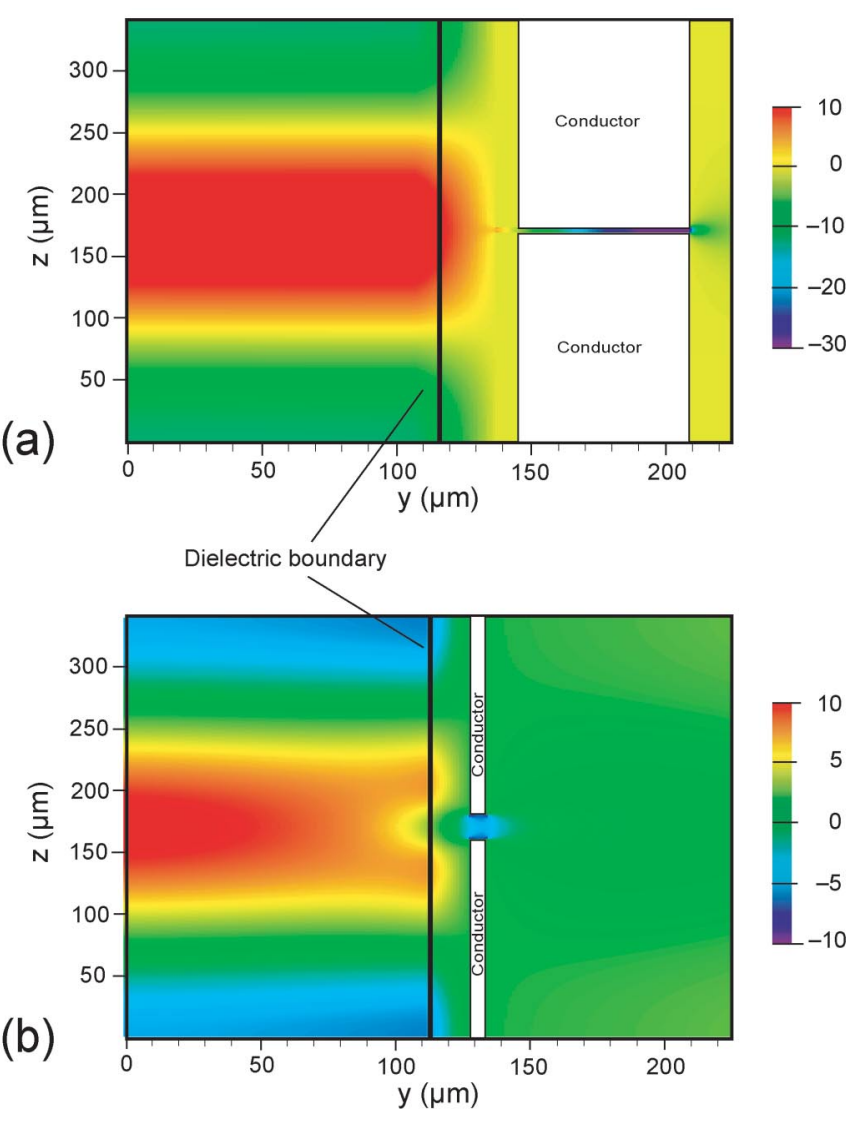

FIG. 5. (Color) False-color map of $E_{z}$ over one structure period in $z$, for slab-symmetric structures with (a) quarter-wave slots, $\Lambda=69 \mu \mathrm{m} \approx \lambda_{g} / 4$, and (b) short slots $\left(\Lambda=5 \mu \mathrm{m} \ll \lambda_{g} / 4\right)$, with dimensions given in Table I. Only the half-plane above the symmetry axis at $y=0$ is shown. Field strengths are in relative units.

time for the 5- $\mu \mathrm{m}$ slots $(150-200 \mathrm{ps})$ is much more realistic for an experiment driven by a short-pulse source such as would be obtained by frequency conversion of a $\mathrm{CO}_{2}$ laser. The shorter pulse duration also reduces the risk of breakdown. Both structures show accelerating fields on the order of $100 \mathrm{MV} / \mathrm{m}$ when driven with a $100 \mathrm{MW}$ laser source focused to a $2 \mathrm{~cm}$ spot.

\section{CONSTRUCTION METHODS AND 3D EFFECTS}

As any physical structure must of course be finite, the full three-dimensional (3D) solution for the fields will depend on the actual horizontal dimensions of the structure. The exact form of the boundary in the long $(x)$ dimension naturally depends on the manufacturing method chosen. We therefore discuss simulations relevant to a 3D structure in combination with possible construction alternatives.

The most natural way to construct any layered, planar device with dimensions in the tens or hundreds of $\mu \mathrm{m}$ is via vapor deposition of the sort used in the semiconductor manufacturing industry. For this reason, our design has 


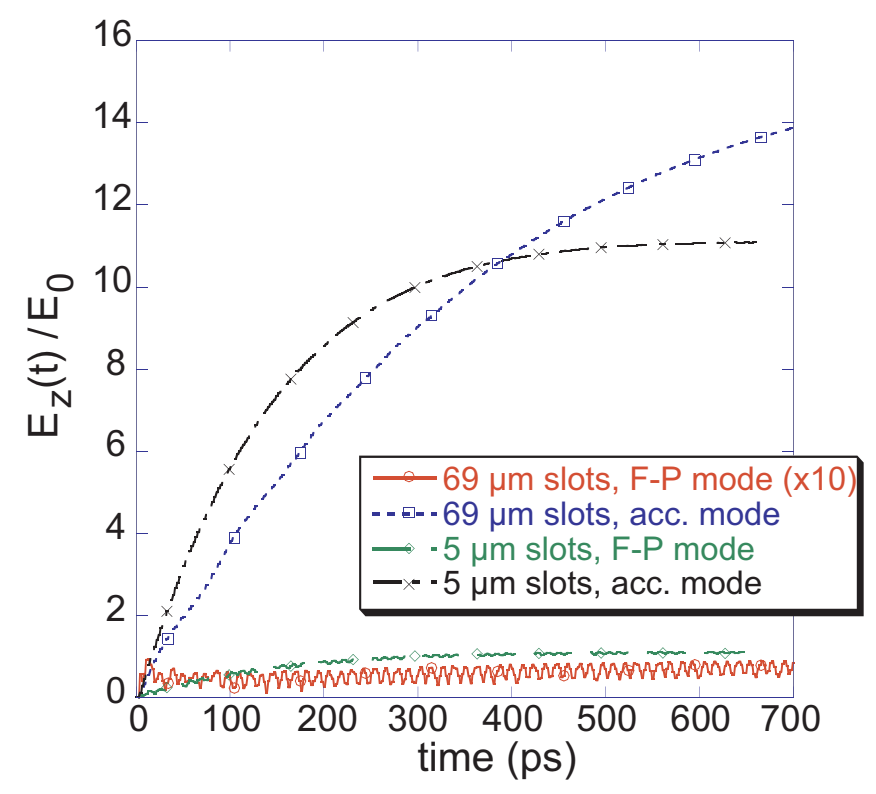

FIG. 6. (Color) Time history of electric field amplitudes for the accelerating (speed-of-light) mode and the Fabry-Perot (zero) mode, comparing the two structure designs from Fig. 5. All fields are normalized to the amplitude of the driving radiation.

used dielectric parameters appropriate to silicon or germanium, both common building materials. Silicon, for example, has a measured (real) dielectric constant of 11.66 to 11.71 at $900 \mathrm{GHz}$, with a loss tangent in this frequency range of $\tan \delta \approx 6-8 \times 10^{-4}$, while germanium has a slightly higher $\epsilon$ and tends to be more lossy [24]. The nonlinear optical response of silicon has been investigated in bulk and thin films [25-27] and at ultrafast time scales [28,29], with the nonlinear dielectric constant $n_{2} I$ having a value in the $10^{-6}$ range at the power levels anticipated, with resulting phase shifts much less than $1 \%$.

One approach to the manufacture of this structure would be to construct the top and bottom dielectric/conductor slabs separately, positioning them correctly relative to each other using micropositioning technology. Such a structure would have open sides, much like a traditional Fabry-Perot resonator, and would need to be considerably wider than the laser spot size in order to limit diffraction of power out of the structure through the sides. In this case, we expect the resonant fields to follow the laser profile in $x$, which we assume to be a Gaussian. One analytical approach to this problem was detailed in Sec. II A, where the field variation was assumed comparable to that of a cosine in the region occupied by the electron beam. If the driving radiation has a purely Gaussian profile, then the $2 \mathrm{D}$ solutions will apply reasonably well to the central region of the accelerator if the laser spot $\sigma_{r}$ is sufficiently large compared to the structure dimension $2 a$. Note, however, that any transverse ( $x$-dimension) mode structure in the laser beam will tend to be imprinted on the cavity fields, which could potentially affect the phase synchronism; clearly, this is an important issue for any future experiment. Simulation of the three-dimensional result in this structure becomes difficult, in part because of the enormous grids required to model the full extent of the fields in the $x$ direction as well as the small coupling slots, and in part because of the poorly defined boundary conditions on $x$. While work continues on this problem, we note that in practice the fields in such a structure are likely to be quite similar to those in the alternate version described in the next paragraph, since a cosinusoidal field variation in $x$ is expected here as well.

An alternate construction method would use a monolithic approach, in which the top and bottom dielectrics are supported by a larger dielectric structure produced through multilayer deposition, as shown in Fig. 7(a). Such a design removes the need for precise external positioning if dimensional tolerances can be made sufficiently small. A partial approach to simulating the fields in this structure can be achieved by using a three-dimensional eigensolver on one period of the structure with coupling slots removed. Figure 7(b) shows the fields obtained by this method; in

TABLE I. Structure dimensions and filling parameters from time-dependent 2D simulation for the two versions of the 340- $\mu \mathrm{m}$ slab-symmetric structure presented in Figs. 5 and 6.

\begin{tabular}{lcc}
\hline \hline \multicolumn{1}{c}{ Parameter } & Quarter-wave slots & Short slots \\
\hline Vacuum gap $(2 a)$ & $230 \mu \mathrm{m}$ & $237 \mu \mathrm{m}$ \\
Dielectric thickness $(b-a)$ & $30 \mu \mathrm{m}$ & $17 \mu \mathrm{m}$ \\
Dielectric relative permittivity $\epsilon$ & 3.0 & 11.69 \\
Slot width $w$ & $5 \mu \mathrm{m}$ & $20 \mu \mathrm{m}$ \\
Slot depth $\Lambda$ & $69 \mu \mathrm{m}$ & $5 \mu \mathrm{m}$ \\
Accelerating field $E_{\max } / E_{0}$ & 15.7 & 11.3 \\
Peak accelerating field $($ per $100 \mathrm{MW}$ drive power) & $122 \mathrm{MV} / \mathrm{m}$ & $89 \mathrm{MV} / \mathrm{m}$ \\
Fabry-Perot mode amplitude & $0.0038 E_{\max }$ & $0.099 E_{\max }$ \\
$1 / e$ filling time $\tau$ & $335 \mathrm{ps}$ & $137 \mathrm{ps}$ \\
$Q=\omega \tau$ & 1850 & 750 \\
Maximum field in slots & $3 E_{\max }$ & $1.3-1.5 E_{\max }$ \\
Frequency detuning $\Delta \omega / \omega_{0}$ & 0 & +0.033 \\
\hline \hline
\end{tabular}


(a)

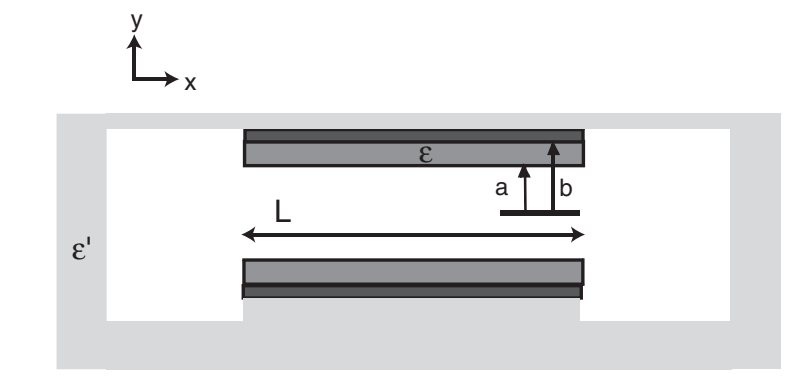

(b)

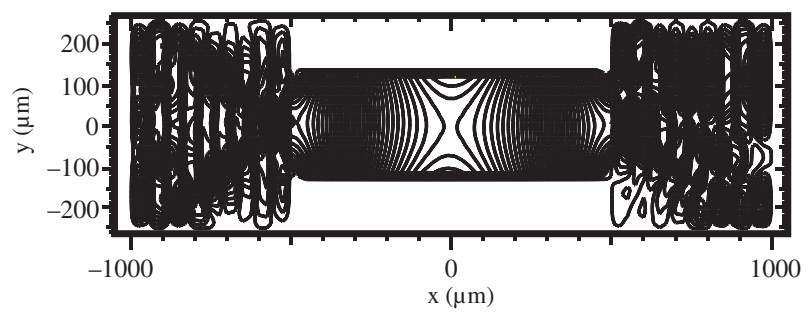

FIG. 7. (a) Geometry of a possible manufacturing approach to the slab-symmetric structure. (b) Accelerating field (from GDFIDL eigenvalue simulation) in such a structure, without coupling slots. The cosinusoidal field dependence in $x$ leads to a slight hyperbolic dependence in $y$.

this case, we see the cosinusoid of the bounded slab structure reappear in the vacuum gap, joined to smallamplitude vibrations in the free-space regions to either side. The considerations of Sec. II A are then valid once more. In principle, it should also be possible to obtain the unperturbed infinite-width fields [Eqs. (1)-(4)] within the vacuum gap, if they are matched to a superposition of cavity modes in the $|x|>L$ regions. We should assume, though, that if there is poor matching at $x= \pm L$, the cosinelike mode will reappear. We further point out that introduction of coupling slots is likely to create field distortions on the order of those observed in Fig. 5.

\section{A. Tolerances}

The manufacturing tolerances allowable for the important structure parameters can be estimated from simulation by working from the frequency shift introduced as parameters are varied. In time-dependent simulation, we derive structure frequency bandwidths on the order of $0.1-0.2 \%$. This corresponds analytically to approximately the same error range on the structure dimensions $a$ and $b$. Varying the slot parameters (width and length) affects both the resonant frequency and the filling factor, but the former with much greater sensitivity. It must be cautioned, however, that drawing rigorous conclusions from a simulation is risky, since at the one-percent level we near the resolution limits of our finite-difference codes. In practice, it will be necessary to have some degree of tunability to the structure, by varying the gap spacing $a$. Active feedback will enable the structure to be tuned to the appropriate frequency, compensating at the same time for mistunings due to the exact experimental parameters. Angular misalignment of the top and bottom dielectric slabs is a more serious problem, and parallelism will need to be maintained at the $0.1 \%$ level.

\section{CONCLUSION AND EXPERIMENTAL OUTLOOK}

In this paper, we have described a slab-symmetric dielectric-loaded structure operating with a novel coupling scheme and in a new wavelength regime. An array of sidecoupling slots, analyzed and simulated for the first time, allows nearly single-mode excitation of the accelerator without significant perturbation of the cavity fields, and with only slight changes in the beam wakefields. The couplers, however, detune the structure and limit the available field; the final design must anticipate these effects. Like other slab-symmetric structures, the resonant laserdriven accelerator described here has advantageous transverse stability for high-charge beams. Unlike previously described structures powered at infrared frequencies, this device is powered by a submillimeter-wave source at 340 $\mu \mathrm{m}$, allowing the structure dimensions to be ample for acceleration of a slab electron beam with realistic transverse size.

When slab-symmetric structures are driven with the high power levels available from optical and submillimeter radiation sources, appreciable accelerating fields may be obtained despite their low shunt impedance. A submillimeter-wavelength radiation source which would be able to drive the structure is under development at UCLA [30]. Utilizing noncollinear mixing of two $\mathrm{CO}_{2}$ laser frequencies in a nonlinear crystal, this source is expected to produce at least $100 \mathrm{MW}$ of power at $340 \mu \mathrm{m}$ wavelength. For the structure described here at these power levels, gradients near $100 \mathrm{MeV} / \mathrm{m}$ are predicted, which should be well below the known breakdown limits for these materials and frequencies. Initially, structure tests may be conducted at much lower powers.

Issues of fabrication technique remain to be resolved before a test structure can be constructed. Experimental investigation of this prototype will begin with low-power cold testing using the output coupling slots to verify the resonant frequency and theoretical dependence on gap spacing. It may also be possible to measure the wakefield radiation spectrum using a test beam. The most significant experimental issue is anticipated to be breakdown limitations, which are not well characterized at present and must be tested by varying the input power density. However, the most common dielectric breakdown mechanism, avalanche ionization, is known to be suppressed at long wavelengths, and is unlikely to be an issue even in the FIR regime.

A successful demonstration of energy gain by a slab beam will depend on detailed knowledge of the physics of these unusual structures. Careful diagnosis of the long- 
wavelength experiment contemplated here may be essential for any future scaling of these devices to the nearoptical range. As such scaling holds the potential to produce accelerating fields of many $\mathrm{GV} / \mathrm{m}$, optical slabsymmetric structures could be an attractive choice for laser-driven charged-particle acceleration.

\section{ACKNOWLEDGMENTS}

Thanks are due G. Travish and P. Schoessow for useful conversations. This work was supported by the U.S. Department of Energy, Grant No. DE-FG03-92ER40693.

[1] J. Rosenzweig, A. Murokh, and C. Pellegrini, Phys. Rev. Lett. 74, 2467 (1995).

[2] C. E. Clayton, K. A. Marsh, A. Dyson, M. Everett, A. Lal, W. P. Leemans, R. Williams, and C. Joshi, Phys. Rev. Lett. 70, 37 (1993).

[3] J. R. Fontana and R. H. Pantell, J. Appl. Phys. 54, 4285 (1983).

[4] Y.C. Huang and R. L. Byer, Appl. Phys. Lett. 69, 2175 (1996).

[5] E. Esarey, P. Sprangle, and J. Krall, Phys. Rev. E 52, 5443 (1995).

[6] A. Tremaine, J. Rosenzweig, and P. Schoessow, Phys. Rev. E 56, 7204 (1997).

[7] J.B. Rosenzweig and P. V. Schoessow, in Advanced Accelerator Concepts, Eighth Workshop, 1998, edited by W. Lawson, C. Bellamy, and D. F. Brosius, AIP Conf. Proc. No. 472 (American Institute of Physics, New York, 1999), pp. 693-700.

[8] P. V. Schoessow and J. B. Rosenzweig, in Proceedings of the 1999 Particle Accelerator Conference, edited by A. Luccio and W. MacKay (IEEE, Piscataway, NJ, 2000), pp. 3624-3626.

[9] Z. Zhang, S. G. Tantawi, and R. D. Ruth, Phys. Rev. ST Accel. Beams 8, 071302 (2005).

[10] C. Wang, J. L. Hirshfield, J.-M. Fang, and T. C. Marshall, Phys. Rev. ST Accel. Beams 7, 051301 (2004).

[11] J. G. Power, W. Gai, and P. Schoessow, Phys. Rev. E 60, 6061 (1999).

[12] L. Xiao, W. Gai, and X. Sun, Phys. Rev. E 65, 016505 (2002).
[13] M.E. Hill, C. Adolphsen, W. Baumgartner, R. S. Callin, X. E. Lin, M. Seidel, T. Slaton, and D. H. Whittum, Phys. Rev. Lett. 87, 094801 (2001).

[14] S. Y. Park, C. Wang, and J.L. Hirshfield, in Advanced Accelerator Concepts, Tenth Workshop, 2002, edited by C. E. Clayton and P. Muggli, AIP Conf. Proc. No. 647 (American Institute of Physics, New York, 2002), pp. 527541.

[15] L. Xiao, C. Jing, W. Gai, and T. Wong, in Advanced Accelerator Concepts, Tenth Workshop, 2002 (Ref. [14]), pp. $542-555$.

[16] L. C. Steinhauer and W. D. Kimura, Phys. Rev. ST Accel. Beams 6, 061302 (2003).

[17] A. Mizrahi and L. Schächter, Phys. Rev. E 70, 016505 (2004).

[18] R. B. Yoder and J. B. Rosenzweig, in Advanced Accelerator Concepts, Eleventh Workshop, 2004, edited by V. Yakimenko, AIP Conf. Proc. No. 737 (American Institute of Physics, New York, 2004), pp. 288-294.

[19] W. Bruns, IEEE Trans. Magn. 32, 1453 (1996).

[20] S. J. Smith and E. M. Purcell, Phys. Rev. 92, 1069 (1953).

[21] K. J. Woods, J.E. Walsh, R. E. Stoner, H. G. Kirk, and R. C. Fernow, Phys. Rev. Lett. 74, 3808 (1995).

[22] J.P. Verboncoeur, A.B. Langdon, and N.T. Gladd, Comput. Phys. Commun. 87, 199 (1995).

[23] D. L. Bruhwiler, R.E. Giacone, J.R. Cary, J.P. Verboncoeur, P. Mardahl, E. Esarey, W. P. Leemans, and B. A. Shadwick, Phys. Rev. ST Accel. Beams 4, 101302 (2001).

[24] J. W. Lamb, Int. J. Infrared Millim. Waves 17, 1997 (1996).

[25] H. K. Tsang, C. S. Wong, T. K. Liang, I. E. Day, S. W. Roberts, A. Harpin, J. Drake, and M. Asghari, Appl. Phys. Lett. 80, 416 (2002).

[26] M. Dinu, F. Quochi, and H. Garcia, Appl. Phys. Lett. 82, 2954 (2003).

[27] G. W. Rieger, K. S. Virk, and J. F. Young, Appl. Phys. Lett. 84, 900 (2004).

[28] K. Sokolowski-Tinten, J. Bialkowski, and D. von der Linde, Phys. Rev. B 51, 14186 (1995).

[29] K. Sokolowski-Tinten, A. Cavalleri, and D. von der Linde, Appl. Phys. A 69, 577 (1999).

[30] P. Musumeci, S. Ya. Tochitsky, C. E. Clayton, C. Joshi, C. Pellegrini, and J.B. Rosenzweig, in Proceedings of the International Conference on Lasers 2001, edited by V. J. Corcoran and T. A. Corcoran (STS Press, McLean, VA, 2002). 